\title{
ARCHIV DER PHARMACIE.
}

4. Band, 2. Heft.

\section{A. Originalmittheilungen.}

\section{Bemerkungen zu der Salzsäure-Reaction bei der Prüfung des Arrow - Root.}

Von Ed. Schaer, Professor in Zürich.

Im Aprilheft des Archivs findet sich eine interessante Notiz von K. Calmberg hinsichtlich des in Pharm. German. beschriebenen Verhaltens der Marantastärke zu verdünnter Salzsäure von 1,09 spec. Gew. (circa $18 \% \mathrm{HCl}$ ). Die daselbst angegebenen negativen Resultate forderten von selbst zu gelegentlichen weiteren Versuchen auf, denn, wenn auch schwerlich irgend eine Pharmacopöe den Anspruch erheben darf, jedes Irrthums in chemischer oder pharmacognost. Beziehung bar zu sein, so mochte doch anzunehmen sein, dass die in der Pharm. Germ. aufgenommene Charakteristik der Arrow Root-Stärke nicht ohne nähere Prüfung des, Materiales und ohne Berücksichtigung der einschlagenden Literatur verfasst worden sei. Ueberdiess vermag in solchen Fragen eine wenn auch noch so unvollständige Versuchsreihe zuweilen etwas zur Aufklärung beizutragen, und glauben wir, die nachstehenden Beobachtungen um so eher mittheilen zu sollen, als die Beobachter bei der Ausarbeitung der Pharm. German. in keinerlei Weise und auch bei deren Gebrauch nur unwesentlich betheiligt sind.

Vorerst muss als erstes Ergebniss einiger vorläufiger Versuche erwähnt werden, dass es in der That, in vollkommener Uebereinstimmung mit den Versuchen Calmbergs, durchaus nicht schwer fällt, aus verschiedenen Apotheken und Arch. d. Pharm. VII. Bds. 2. Hft. 
Droguerien Arrow - Root zu beschaffen, welches, bei mikroskop. Prüfung unzweifelhaft als Maranta-Stärke charakterisirt, mit Salzsäure im Sinne der Pharmacopöe behandelt sehr rasch eine vollständige fast durchscheinende Gallerte (ohne Krautoder Bohnengeruch) liefert, ebenso wie es andrerseits leicht gelingt, Proben zu erhalten, die, gleichfalls als ächt erkannt, sich sehr genau nach den Angaben der Pharm. verhalten, d. h. bei 10 Minuten langem Agitiren mit Salzsüure sich nicht verdicken und auch bei längerer Einwirkung in der Kälte $\left(15^{\circ} \mathrm{C}\right.$.) nur spärlich gelöst werden.

Diese mit früheren gelegentlichen Beobachtungen übereinstimmenden Erfahrungen veranlassten eine eingehendere Versuchsreihe mit 13 Stärke-Arten, worunter 11 Proben von Arrow-Root, welche theilweise der hiesigen pharmacognost. Sammlung (Eidg. Polyt.) entnommen, theilweise von renommir. ten deutschen Droguenhandlungen freundlichst und mit besonderer Sorgfalt besorgt worden waren. Die nachstehende Liste enthält zunächst die Bezeichnung resp. Signatur der einzelnen Sorten, sodann, in Klammer beigefügt, die botanische Abstammung, die für jede Nummer mit aller Vorsicht und unter Mithülfe der besten pharmacognost. und botan. Abbildungen bestimmt wurde. Dass hierbei Proben, die mit anorgan. Beimengungen verunreingt waren oder ein Gemenge zweier oder mehrerer Stärkearten darstellten, gänzlich unberücksichtigt blieben, darf als selbstverständlich vorausgesetzt werden.

a) Kartoffelstärke (ächt von Solanum).

b) Weizenstärke (ächt von Triticum).

c) Brasilian. Arrow - Root (Manihot).

d) Maranta - Stärke (Maranta).

e) Arrow - Root v. Port Natal (Maranta).

f) Arrow - Root, westindisch (Maranta).

g) Arrow-Root v. Bermudas (Manihot).

h) Arrow-Root $\nabla$. Jamaica (Maranta).

i) Arrow-Root v. Parkhill (Maranta).

k) Ostindisches Arrow - Root (Manihot).

1) Arrow-Root v. St. Vincent (Maranta). 
m) Indisches Arrow-Root, Tikhar (Curcuma).

n) Maranta Arrow - Root aus Java (Maranta).

Mit diesen Proben wurde die Salzsäure-Reaction genau nach Angabe der deutschen Pharmacopöe vorgenommen und verhielten sich dabei die vorstehenden, nach ihrer Veränderung durch das besagte Reagens geordneten Arrow-Root-Marken folgendermaassen;

a) in wenigen Minuten dicke, beinahe klare Gallerte bildend, nach einigen Stunden vollkommene Lösung (sehr prägnanter Kraut-oder Bohnengeruch);

b) keine Gallerte bildend, erst nach einigen Stunden stark opalisirende Lösung;

c) verhält sich wie a;

d) nach 5 Minuten dicke trübe Gallerte liefernd, allmählig sich verflüssigend;

e) verhält sich wie $d$;

f) nach 10 Minuten dickliche, trübe Gallerte liefernd, darauf bald dünnflüssig;

g) verhält sich wie d und e;

h) nach 10 Minuten nicht gallertartig, sondern zum grössten Theile pulverförmig abgeschieden, auch nach 24 Stunden und länger nur partioll gelöst;

i) verhült sich wie $h$;

k) verhält sich wie $h$;

1) verhält sich wie $h$;

m) verhält sich wie $h$;

n) verhält sich wie $h$.

Es ergiebt sich nun aus den vorstehend mitgetheilten Ergebnissen, deren chemischer Theil in $\mathrm{m}$. Laboratorium durch H. Pharmaceuten Th. Küpfer ausgefiihrt wurde, zunächst die Thatsache, dass Manihot-Stärke gegenwärtig nicht allein als "brasilian. Arrow-Root", sondern auch als "ostindisches" Arrow-Root, *) ja sogar unter der Signatur des als Marantastärke hochgeschätzten Bermuda-Arrow Root, im Handel getrof-

*) Siehe über Manihot-Cultur in Indien Flückigers Lehrbuch und Pharmacognosie 1867. p. 713. b. Amyl. Marantae. 
fen wird. Zweitens aber werden wir darüber belehrt, dass sowohl das Amylum von Manihot als dasjenige von Maranta unter besondern, für mich noch räthselhaften Umständen ein durchaus verschiedenes Verhalten zu Salzsäure zeigen kann. In der That finden wir nach den obenstehenden Tabellen, dass unter 7 Amylumproben, die bei genauer Untersuchung sämmtlich unzweifelhaft als von Maranta stammend erkannt wurden, drei Proben sich von der Pharmacopöe verschieden, dagegen mit Calmbergs Angaben übereinstimmend verhielten, während die vier übrigen Marken in der Salzsäurereaction durchaus conform mit der Charakteristik der Pharmacopöe blieben. Muss nun auch zugegeben werden, dass die in Frage stehende Reaction, nach unserer Versuchsreihe zu urtheilen, mindestens ebenso berechtigt erscheint, als etwa die entgegenstehende Angabe, dass Maranta-Arrowroot mit Salzsäure gelatinire und dass ausserdem die von der Pharm. Germ. vertretene Ansicht noch durch die, gleichfalls auf sorgfältiger Beobachtung beruhenden Angaben in der neuen, classischen "Pharmacographia" von Flückiger und Hanbury (s. pag. 571 und 573) gestützt wird, so ist andrerseits doch der Schluss nicht ungerechtfertigt, dass das Verhalten des Arrow-Roots zu Salzsäure nicht als einfaches und sicheres Mittel zur Identificirung des Maranta-Amylums dienen kann, sondern dass bei dieser Reaction höchstens der characterist. Geruch, der bei Vermengung oder Substitution mit Kartoffelstärke zu beobachten ist und der, wie ich nach zahlreichen Versuchen bestätigen kann, nur bei der Stärke von Solanum tuberosum auftritt, als maassgebend betrachtet werden darf.

Es mag hierbei noch erwähnt werden, dass die zwischen $15-40^{\circ}$ vor sich gehende Einwirkung einer Salzsäure von 1,06-1,09 spec. Gew. auf die hierbei gallertartig werdenden Amylumarten dem Verhalten der Stärke zu Brom - oder Jodkalium, Chlorcalcium, Natriumnitrat und ähnl. leicht löslichen Salzen - wie solches in dem oben angeführten Handbuche von Flückiger und Hanbury, noch ausführlicher aber in Flückigers Lehrbuch dor Pharmacognosie in dem monographisch gehaltenen Artikel: Amylum Marantae beschricben wurde 
- in vielfacher Beziehung sehr ähnlich ist. In beiden Fällen bemerken wir zunächst ein starkes Aufquellen des Stärkekorns, allmählig gefolgt von wirklicher Lösung und daheriger Verflüssigung der anfänglich unter gewissen Umständen gallertförmigen Masse; in beiden Fällen werden die Löslichkeitsverhältnisse des Amylums zu Wasser eigenthümlich verändert, insofern auch bei starker Verdïnnung der salzbaltigen oder säurehaltigen Amylumlösung eine klare Flüssigkeit resultirt, aus welcher durch Alkohol strukturloses Amylum mit seinen vorherigen chemischen Eigenschaften gefällt werden kann. Dass hierbei die Löslichkeit nicht durch Uebergang in Dextrin und Zucker bedingt ist, wie diess schon von Flückiger nachgewiesen wurde, kann nach $\mathrm{m}$. Beobachtung besonders auch bei der Quellung und Lösung verschiedener Stärkearten in Salzsäure (1,06-1,08 sp. Gew.; $20-30^{\circ}$ Temp.) sehr deutlich constatirt werden; die Gegenwart der eben genannten Körper ist in der ersten Periode nicht nachweisbar und erfolgt, im Gegensatz zu der Säurowirkung bei der Siedehitze, sehr langsam, so dass nach langer Zeit noch unverändert gelöstes Amylum durch die blauviolette Färbung (nicht Fällung) der Lösung mittelst Jodwasser erkennbar bleibt. Die Analogie im Verhalten des Amylums zu officineller Salzsäure und zu den angeführten leichtlöslichen Substanzen (zu welchen auch Chloralhydrat gehört), geht u. A. aus dem weitern Umstande hervor, dass von den oben besprochenen Amylumproben nur diejenigen, welche mit Salzsäure Gallertbildung und nachherige klare Lösung zeigten, auch in Contact mit Jodkalium - oder Chlorcalciumlösungen zu vollkommener Quellung und leichter gänzlicher Aullösung gelangten, wobei in dem einen und andern Falle nur äusserst kleine Mengen der Substanz in Form jener zarten Flocken zurückblieben, die, durch Jodlösung nur röthlich oder deutlich blau gefärbt, von Nägeli und Anderen als Rudimente des Cellulose - Gerüstes des Stärkekornes betrachtet werden, einigermaassen deutlicher jedoch bei der saccharificirenden Wirkung einiger Fermente auf das Amylum erkennbar sind. Hinsichtlich der physikalisch - chemischen Berechtigung dieser Anschauung über 
die Stärke kann ich mich, insoweit die Beobachtungen iuber die Einwirkung verd. Salzsäure Beweiskraft besitzen, nur zu den bekannten Ansichten, Flückigers bekennen.

Soll schliesslich noch der Ursache gedacht werden, welche dem so auffallend abweichenden Verhalten verschiedener Marantastärke-Proben zu Salzsäure zu Grunde liegt, so muss leider bekannt werden, dass keiner der hierauf bezüglichen Versuche zu befriedigender Erklärung geführt hat. Namentlich scheint der durch verschiedenen Wassergehalt bedingte Unterschied im specif. Gewichte, den zuerst Flückiger durch klar beweisende Untersuchungen erläuterte, und in dem ich anfänglich den Grund des verschiedenen Ausfalls der Salzsäure Reaction vermuthete, ohne Einfluss zu sein. Obwohl die gewiegtesten Kenner der Stärke, so namentlich Flückiger in dem Nachtrage seines Lehrbuches keine nähern Angaben über Verschiedenbeit wasserhaltigen lufttrockenen und wasserfreien Amylums be $i$ Berührung mit Haloidsalzen oder verd. Salzsäure mittheilen, schien es doch im Hinblick auf die widersprechenden, beiderseits richtigen Angaben in der Pharmacop. Germ. und der Notiz Calmbergs geboten, sich nach dem spec. Gewichte der 13 citirten Versuchsobjekte umzusehen. Die Prüfung der unter sehr annähernd gleichen Umständen aufbewahrten Proben geschah nach der 1. Zeit durch Flückiger angegebenen einfachen, aber für diese Zwecke ausreichenden Weise mit Chloroform reinster Beschaffenheit. Sämmtliche Proben schwammen auf Chloroform und blieben theilweise suspendirt; keine einzige zeigte ein Untersinken und so bewegte sich daher das spec. Gew. aller geprüften Stärkearten innerhalb sehr nahe an 1,50 liegender Grenzen, da schon bei 1,55 spec. Gew. deutliches Sinken in Chloroform stattfindet; ausserdem zeigten zwei Proben (aus obiger Tabelle), beide als ächte Marantastärke erkannt, von denen die eine mit Salzsäure sich rasch verdickte, während die zweite sich indifferent verhielt, nach sehr sorgfältiger, vollkommener Trocknung bei $100^{\circ}$ einen Gewichtsverlust von 12,6 und 13,0 Procent, und es ist daher anzunehmen, dass diese geringfügige Differenz im Wassergehalte der Iufttrocknen Pro- 
ben mit dem Verhalten zur Säure in Verbindung stehen möchte. Es bleibt daher, wie mir scheint, bis zu späterer Auflösung wohl nur die Vermuthung übrig, dass Veränderung klimat. Einflüsse oder wahrscheinlicher Unterschiede dor Bereitungsweise gewisse minime Verschiedenheiten der StärkeStruktur oder Aenderungen der normalen Beschaffenheit bedingen, wodurch zwar nicht die wichtigen Eigenschaften, einschliesslich der Fähigkeit der Wasseraufnahme, wohl aber das Verhalten zu einigen lösenden Agentien modificirt wird.

Zürich, Juni 1875 .

\title{
Notiz über die Löslichkeit des Bittermandelöles in Wasser.
}

\author{
Von F. A. Flü ckiger.
}

In den meisten chemischen Lehrbüchern und Nachschlagewerken wird angegeben, dass 30 Theile Wasser $1 \mathrm{Th}$. littermandelöl aufzulösen vermöchten. Ich finde dieses nicht bestätigt, weder für gewöhnliches blausäurehaltiges, noch für blausäurefreies Oel, noch auch für solches, ebenfalls blausäurefreies $\mathrm{Oel}$, welches aus einer krystallisirten Bisulfitverbindung $2\left(G^{7} \mathrm{H}^{6} \theta, \mathrm{NaHS}^{3}\right)+\theta \mathrm{H}^{2}$ vermittelst kohlensauren $\mathrm{Na}-$ trons im Kohlensäurestrom abgeschieden wurde, um Benzoësäure zu beseitigen, welche vorhanden sein konnte. Keine Rede davon, dass 30,50,100, 200 Theile Wasser einen Theil des einen oder andern Oeles aufzunehmen im Stande sind. Erst bei etwa dem 250 fachen Gewichte Wasser beginnen die schweren Oeltropfen zu verschwinden, d. h. sie vertheilen sich sehr fein in dem Wasser und verleihen ihm cin trübes Aussehen. Bei 300 Theilen Wasser wird das Gemisch merklich klarer, aber selbst bei weit mehr Wasser nicht völlig klar. Es ist nicht wohl möglich, das Verschwinden der Trübung scharf zu beobachten, weil es nur sehr allmählig eintritt. In der Praxis wird überdies die Löslichkeit noch ferner beeinflusst durch die Bildung von Benzoësäure und von Hydrobenzamid, welche beide in kaltem Wasser 